\title{
CP Violations Newly Observed in Beauty Meson Decays
}

\author{
Measurements show large matter-versus-antimatter differences in three-pion decays of $B$ \\ mesons, yielding new insights into the strong interaction dynamics that control these decays.
}

\section{by J. Michael Roney*}

$\mathrm{P}$ hysicists are engaged in a world-wide program of probing the intriguing, and rare, phenomenon of $C P$ violation - a phenomenon needed to explain the dominance of matter over antimatter in the Universe [1]. As part of this program, the $\mathrm{LHCb}$ experiment at CERN reported in 2014 that the $B^{-}$meson-a negatively charged particle containing a beauty (or bottom) quark-decays to three pions in a way that is different than how its antimatter counterpart, the $B^{+}$meson, decays [2]. However, these measurements did not yield the sources of $C P$ violation, which remained hidden-like a "diamond in the rough"-by all the complexities of strong force dynamics in these decays. With more than a fourfold increase in its data set, $\mathrm{LHCb}$ now reports three separate sources of $C P$ violation within three-pion decays and provides key insights into the strong interaction dynamics [3]. Those insights may ultimately help determine if there is a new source of $C P$ violation-one that goes beyond current models and can explain the matter-

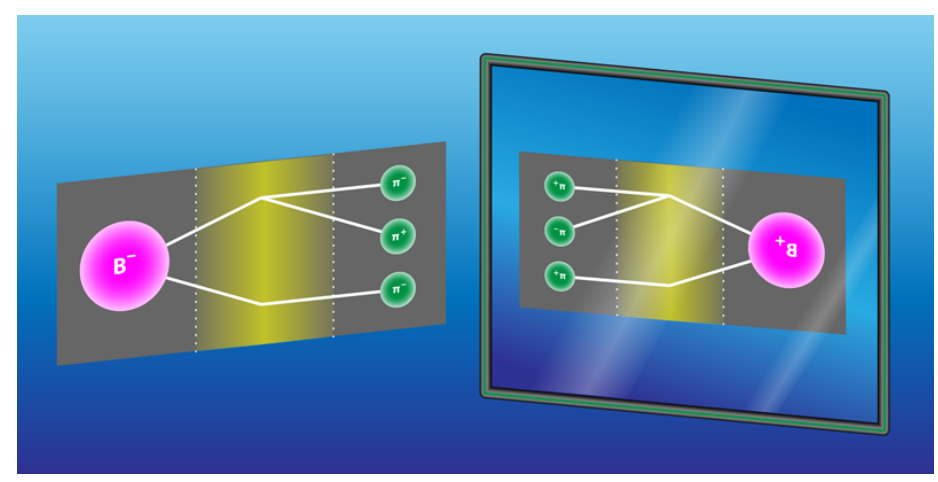

Figure 1: The CP symmetry can be thought of as a "mirror" whose image also converts matter to antimatter. New experimental observations show that this symmetry is violated by the decay of charged $B$ mesons into three pions. (APS/Alan Stonebraker)

*Department of Physics and Astronomy, University of Victoria, Victoria, BC, Canada antimatter asymmetry of our Universe.

Violations of $C P$ symmetry may sound like a breakdown in theory, but they are in fact an integral part of the standard model of particle physics. The $C$ of $C P$ refers to the "charge conjugation" operation in which every particle in a process is replaced by its antiparticle equivalent. The $P$ refers to "parity transformation," which inverts the spatial coordinates: $(x, y, z) \rightarrow(-x,-y,-z)$. Processes associated with the strong and electromagnetic forces behave identically under $C$ or $P$ transformations. However, the weak interaction is not symmetric under $C, P$, or the combination $C P$ (Fig. 1).

$C P$ violation was first observed [4] in 1964 by James Cronin and Val Fitch and their collaborators at Brookhaven National Laboratory in New York, and it led to their 1980 Nobel Prize in Physics. The measurements showed a difference, or $C P$ asymmetry, of $0.2 \%$ in the weak interaction decays of neutral $K$ mesons, depending on whether they contained a strange quark or strange antiquark. In order to explain this matter-antimatter disparity, Japanese theorists Makoto Kobyashi and Toshihide Maskawa (KM) postulated in 1973 [5] that nature has six quarks, rather than the three known to exist at the time. The KM model assumes that the six quarks mix in such a way that strange-quark processes differ from strange-antiquark processes-thus explaining the observed $C P$ violation in $K$ mesons.

The KM theory also predicted large $C P$ violation in some rare decays of particles containing beauty quarks, with $C P$ asymmetries as high as $70 \%$. By 2001, the BaBar experiment [6] at SLAC in California and the Belle experiment [7] at KEK in Japan observed the predicted $C P$ violation in the decays of neutral $B$ mesons and anti- $B$ mesons decaying to the same final state. This led to the 2008 Nobel Prize in Physics for Kobyashi and Maskawa and the firm embedding of the KM theory within the standard model. However, the $\mathrm{KM}$ mechanism for producing $C P$ violation was found to be several orders of magnitude too small to explain the matter domination in the Universe. Thus researchers have been on the lookout for sources of $C P$ violation beyond the standard model.

This search for "extra" CP violation is an important motivation for $\mathrm{LHCb}$, which studies the particles that stream out of high-energy proton-proton collisions at the Large Hadron 
Collider (LHC). The LHCb experiment consists of multiple particle detectors close to the axis of the LHC's proton beam lines where $B$ mesons, as well as other mesons containing charm quarks, are produced and quickly decay. With the enormous numbers of events being recorded, LHCb is sensitive to $C P$ violation in very rare decays of both $B$ and charm mesons. In fact, $\mathrm{LHCb}$ reported the first observation of $C P$ violation in charm decays last year [8] (see Viewpoint: Charm Reflects Poorly on Anticharm).

When it comes to the three-pion decays of charged $B$ mesons-written as $B^{+} \rightarrow \pi^{+} \pi^{-} \pi^{+}$and $B^{-} \rightarrow$ $\pi^{+} \pi^{-} \pi^{-}-C P$ violation is expected, but the exact amount is not well predicted. The uncertainty stems from the difficulty in calculating the strong interactions between the mesons. This strong interaction dynamics produces a range of different intermediate states (or decay modes), which each have a different $C P$ asymmetry. Previous experiments, such as the BaBar experiment, have studied these three-pion decays and found features potentially related to $C P$ violation [9]. However, because the process is so rare, BaBar did not have enough data to actually see the $C P$ violation. With over $20,000 B^{+} \rightarrow \pi^{+} \pi^{-} \pi^{+}$decays in hand, LHCb has 17 times the size of the BaBar experiment's decay sample. That provides enough statistics to not only observe $C P$ violating effects but to also test and validate models of the behavior of the strong interaction in these decays for the first time.

What the $\mathrm{LHCb}$ team observes is fascinating, as they are able to observe the different ways that $B$ mesons can decay to three pions. For example, the $B$ meson can initially decay into an intermediate state consisting of a single pion and a spin- 1 rho meson $\left(\rho^{0}\right)$ with a mass of $770 \mathrm{MeV} / c^{2}$, which itself decays to a $\pi^{+} \pi^{-}$pair. This quasi-two-body decay is the dominant mode, accounting for $55 \%$ of the threepion decays. LHCb also observed other modes, such as one where the pion pair comes through a spin-2 meson-called $f_{2}(1270)$ - with a mass of $1270 \mathrm{MeV} / c^{2}$, and another mode in which the pair exists in a spin-0 state with an effective mass below the rho mass (Fig. 2). The LHCb team used these results to determine the parameters that go into models of the strong interaction dynamics of this decay [10].

The collaboration observed $C P$ violation-with a significance of more than 10 sigma-in two of the three-pion decay modes: the mode with $f_{2}(1270)$ exhibited a $C P$ asymmetry of $40 \%$, whereas the spin- 0 decay mode had a $15 \%$ asymmetry. $\mathrm{LHCb}$ also established the presence of $C P$ violation effects that it attributes to interference between the spin-0 and spin-1 states. However, the data showed no significant $C P$ violation in the dominant decay mode with the spin-1 rho meson.

The LHCb observations of $C P$ violation in these $B$ meson decays are entirely consistent with predictions of the standard model. Consequently, this work doesn't address the problem of the matter-dominated Universe. However, by providing experimental constraints and reliable models of
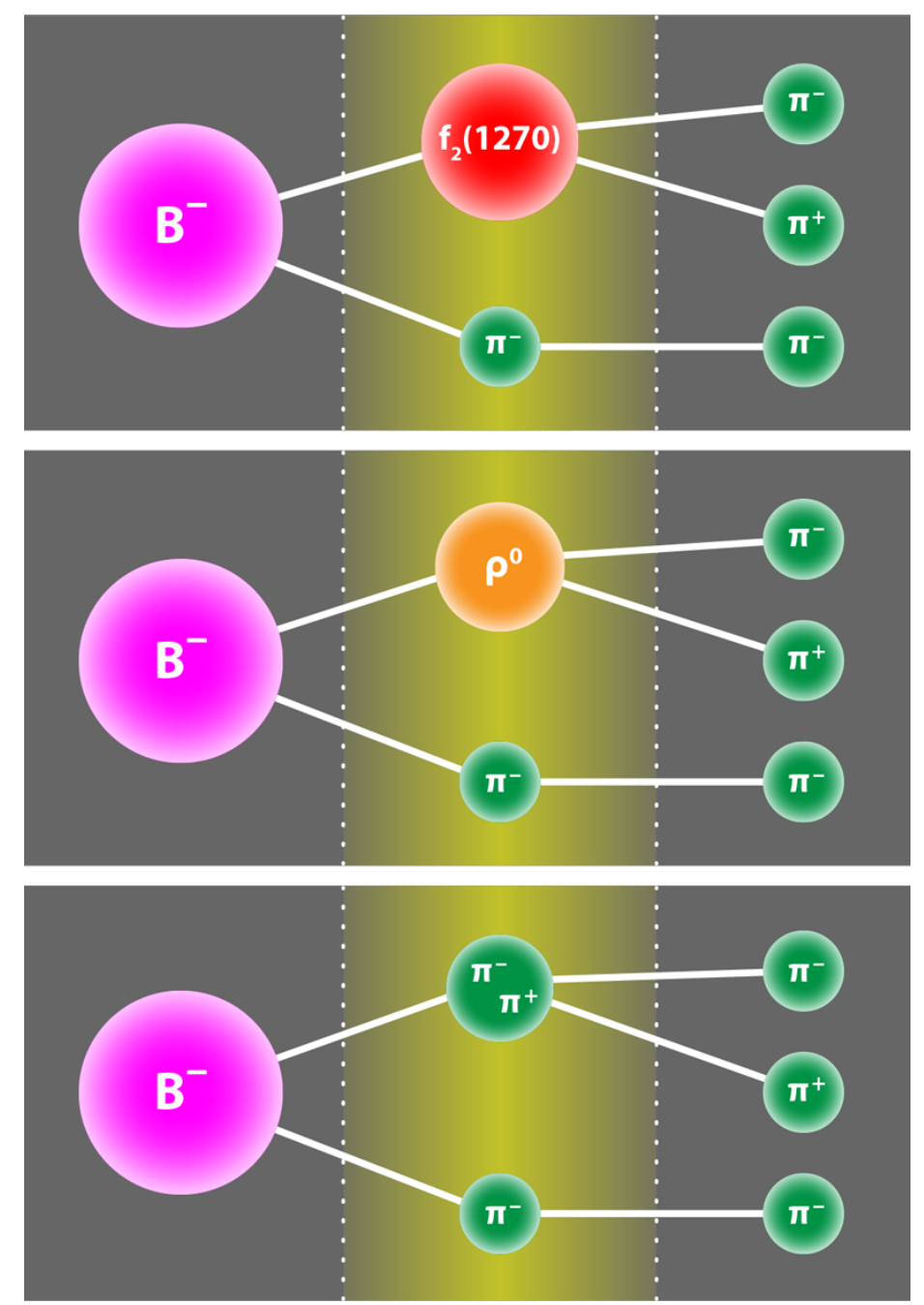

Figure 2: The decay of charged $B$ mesons into three pions occurs through several different intermediate states, including the spin-2 $f_{2}(1270)$ meson (top), the spin-1 rho meson (middle), and a spin-0 double-pion state (bottom). (APS/Alan Stonebraker)

the strong dynamics of charge- $B$-to-three-pion decays, these $\mathrm{LHCb}$ results can be used to reduce the uncertainties in the extraction of $\mathrm{CP}$-violating observables in other related decays, such as decays of neutral $B$ mesons to three pions $\left(B^{0} \rightarrow \pi^{+} \pi^{-} \pi^{0}\right.$ and anti- $\left.B^{0} \rightarrow \pi^{+} \pi^{-} \pi^{0}\right)$, which will be used to directly test the KM model with higher precision. With those reduced uncertainties, future precision measurements of such decays at $\mathrm{LHCb}$ or at the newly commissioned Belle II in Japan might uncover the most prized diamond in the rough: a new source of $C P$ violation that lies beyond the standard model.

This research is published in Physical Review Letters and Physical Review D. 


\section{REFERENCES}

[1] A. D. Sakharov, "Violation of $C P$ invariance, $C$ asymmetry, and baryon asymmetry of the universe," Usp. Fiz. Nauk 161, 61 (1991); Sov. Phys. Usp. 34, 392 (1991).

[2] R. Aaij et al. (LHCb Collaboration), "Measurement of $C P$ violation in the phase space of $B^{ \pm} \rightarrow K^{+} K^{-} \pi^{ \pm}$and $B^{ \pm} \rightarrow$ $\pi^{+} \pi^{-} \pi^{ \pm}$decays," Phys. Rev. Lett. 112, 011801 (2014).

[3] R. Aaij et al. (LHCb Collaboration), "Observation of several sources of $C P$ violation in $B^{+} \rightarrow \pi^{+} \pi^{+} \pi^{-}$decays," Phys. Rev. Lett. 124, 031801 (2020).

[4] J. H. Christenson et al., "Evidence for the $2 \pi$ decay of the $K_{2}^{0}$ meson," Phys. Rev. Lett. 13, 138 (1964).

[5] M. Kobayashi and T. Maskawa, "CP-violation in the renormalizable theory of weak interaction," Prog. Theor. Phys. 49, 652 (1973).
[6] B. Aubert et al., "Observation of CP violation in the $B^{0}$ meson system," Phys. Rev. Lett. 87, 091801 (2001).

[7] K. Abe et al. (Belle Collaboration), "Observation of large CP violation in the neutral B meson system," Phys. Rev. Lett. 87, 091802 (2001).

[8] R. Aaij et al. (LHCb Collaboration), "Observation of $C P$ violation in charm decays," Phys. Rev. Lett. 122, 211803 (2019).

[9] B. Aubert et al. (BaBar Collaboration), "Dalitz plot analysis of $B^{ \pm} \rightarrow \pi^{ \pm} \pi^{ \pm} \pi^{\mp}$ decays," Phys. Rev. D 79, 072006 (2009).

[10] R. Aaij et al. (LHCb Collaboration), "Amplitude analysis of the $B^{+} \rightarrow \pi^{+} \pi^{+} \pi^{-}$decay," Phys. Rev. D 101, 012006 (2020).

[11] Y. Li et al., "Quasi-two-body decays $B_{(s)} \rightarrow P f_{2}(1270) \rightarrow P \pi \pi$ in the perturbative QCD approach," Phys. Rev. D 98, 056019 (2018).

10.1103/Physics. 13.6 